\title{
Lewisian quidditism, humility, and diffidence
}

\author{
Benjamin L. Curtis ${ }^{1}$
}

(C) The Author(s) 2016. This article is published with open access at Springerlink.com

\begin{abstract}
In 'Ramseyan Humility' Lewis presents the Permutation Argument for quidditism. As he presents it the argument is simple enough, but once one digs beneath its surface, and attempts to understand it in strictly Lewisian terms, difficulties arise. The fundamental difficulty is that, as he presents it, the argument only seems to be sound if one rejects views that Lewis explicitly holds. One aim of this paper is to clarify the argument to show that one can make sense of it in strictly Lewisian terms. In so doing I clarify Lewis's view, clearly lay out the commitments that Lewis has, and define quidditism in strictly Lewisian terms. However, I also have a secondary aim. Lewis accepts that quidditism entails a form of scepticism, that he calls 'Humility'. However, by an extension of the permutation argument I show that quidditism entails a form of scepticism, that I call 'Diffidence', that is far more wide-reaching than Humility.
\end{abstract}

Keywords Metaphysics · Quidditism · David Lewis · Properties · Possibility

\section{Introduction}

In 'Ramseyan Humility' Lewis presents the Permutation Argument for quidditism. As he presents it the argument is simple enough, but once one digs beneath its surface, and attempts to understand it in strictly Lewisian terms, difficulties arise. The fundamental difficulty is that, as he presents it, the argument only seems to be sound if one rejects views that Lewis explicitly holds. One aim of this paper is to clarify the argument to show that one can make sense of it in strictly Lewisian

Benjamin L. Curtis

Benjamin.curtis@ntu.ac.uk

1 Nottingham Trent University, Nottingham, UK 
terms. In so doing I clarify Lewis's view, clearly lay out the commitments that Lewis has, and define quidditism in strictly Lewisian terms. However, I also have a secondary aim. Lewis accepts that quidditism entails a form of scepticism, that he calls 'Humility'. However, by an extension of the permutation argument I show that quidditism entails a form of scepticism, that I call 'Diffidence', that is far more wide-reaching than Humility.

In Sect. 1 I outline the technical terms needed to understand the Permutation Argument and show how its conclusion leads to Humility. In Sect. 2 I give the Permutation Argument as Lewis presents it. In Sect. 3 I raise difficulties for that presentation. In Sect. 4, I restate the argument in strictly Lewisian terms before extending that argument in Sect. 5 to show how quidditism also leads to Diffidence.

\section{Preliminaries}

Humeanism, roughly, is the view that there are no necessary connections between distinct existences. Quidditism, roughly, is the view that properties are distinct elements of possibilities. Lewis holds both views-he is a Humean quidditist. Humean quidditism, Lewis maintains, entails a putatively ominous form of scepticism according to which we are irremediably ignorant about the fundamental properties that feature in the laws of nature that govern our world. We can know that fundamental property roles are uniquely occupied, but it is in principle impossible to know which properties uniquely occupy them. (Or to put it in semantic terms, we can know that our fundamental property terms refer, but we cannot know which properties they refer to.) Lewis calls this form of scepticism 'Humility', and embraces it. 'Why is Humility “ominous"?', he writes, 'Who ever promised me that I was capable in principle of knowing everything?' (Lewis 2009, p. 211) Lewis's unconcern is due to the fact that if we confidently assume that the theoretical terms of our best physics (or perhaps some future physics) refer-i.e. that the fundamental property roles described by physics are indeed uniquely occupied-Humility cannot undermine that confidence. If we are Humble, we can still know that our fundamental property terms refer, and Lewis thinks that this is enough to be getting along with.

If Humeanism is true then possibility is governed by a principle of Recombination according to which we obtain possibilities by taking apart and putting together the distinct elements of possibilities. If quidditism is true then fundamental properties are, in Sellers's nice phrase 'as substitutable for one another in any context as pennies' (Sellars 1948, p. 300). They are featureless fundamental property role-fillers that have no nature (i.e. no essential second-order properties are attributable to them). The Permutation Argument draws on these features of Humeanism and quidditism to show that, taken jointly, these views entail the following proposition that leads to Humility:

M1: If the actual world is nomologically well-behaved then there exist distinct worlds that are nomologically and observationally isomorphic with the actual world. 
To see that M1 leads to Humility first consider some theory $T$ of a world $W$. Suppose that this theory is complete, viz. that it correctly describes all of the fundamental property roles present in $W{ }^{1} T$ is the set of all the logical entailments of the postulate of $T$, viz. a sentence of the form $\mathrm{T}(t 1 \ldots t n)$ where $t 1 \ldots t n$ are the theoretical terms of $T$. Replacing $t 1 \ldots t n$ in the postulate with variables $x 1 \ldots x n$ gives us an open sentence, the realization formula of $T$, that can be satisfied by $n$-tuples of fundamental properties. If, in addition to being complete, $T$ is true, then the realization formula of $T$ will be uniquely satisfied by some n-tuple of fundamental properties, the (unique) realization of $T$, and the sentence obtained by prefixing the realization formula by $n$ existential quantifiers, the Ramsey sentence of $T$, will therefore also be true. ${ }^{2}$

To say that a world is nomologically well-behaved is to say that one property, and one property only, occupies each of the fundamental property roles present in that world and that no property occupies more than one role. So in all nomologically well-behaved worlds some complete theory $T$ is true, so the realization formula of $T$ is uniquely satisfied, and so the Ramsey sentence of $T$ is true. To say that a world is nomologically isomorphic with some well-behaved world $W$ is to say that exactly the same fundamental property roles are present in it as in $W$ and that the very same realization formula that is uniquely satisfied in $W$ is uniquely satisfied in it. So, any world that is nomologically isomorphic with a nomologically well-behaved world $W$ is also nomologically well-behaved and the very same Ramsey sentence that is true at $W$ is true at it.

To say that a world is observationally isomorphic with another world $W$ is to say that for every possible observer in it, there is a possible observer in $W$ to whom everything seems the same, and so no possible observer could tell the difference between it and $W$. If, per impossibile, an observer were to instantaneously swap places with a corresponding observer in an observationally isomorphic world, he/ she would not (and could not) notice any difference. ${ }^{3}$ One important point to note

\footnotetext{
${ }^{1}$ NB A complete theory correctly describes the fundamental property roles in a world iff it has the right balance of strength and simplicity and enables one, in principle, to predict all observable phenomena in a world (at least insofar as it is possible to do so- a complete theory might be probabilistic). But this does not make it true, at least not on Lewis's view. Whether it is true depends on whether the fundamental property roles it specifies are uniquely filled. A complete theory on Lewis's view is thus one that would be true if nature had played its part, and a fundamental property role is a role in a complete theory that would be uniquely filled if nature had played its part (where this condition is met trivially in the case where nature does play its part).

2 For more on theories, postulates, realization formulas, and Ramsey sentences see Lewis (1970, 2009).

3 Cf Black (2000) where he considers a world that is distinct from ours but is (in my terms) observationally and nomologically isomorphic: 'Let us start by considering the world isomorphic with ours... To the inhabitants of such a world, their world looks just like ours, but in reality it is supposed to be quite different.' (p. 94). The definition of observational isomorphism that I have given, referring to possible observers as it does, is not as clear as I would like. But I think it is clear enough and therefore serviceable. I would like to have defined the notion in a weaker but clearer way as follows: A world $W x$ is observationally isomorphic with another $W y$ iff for all observers $o x$ in $W x$, there exists an observer oy in $W y$ such that everything seems the same to $o x$ as it does to $o y$, and for all observers $o y$ in $W y$, there exists and observer $o x$ in $W x$ such that everything seems the same to $o y$ as it does to $o x$. (This definition is weaker than the original definition because, as all actual observers are possible observers, it is entailed by but does not entail it.) And in fact I think that, strictly speaking, this weaker definition is all that I need.
} 
here is that, for Lewis, worlds in which the same Ramsey sentence are true are ipso facto observationally isomorphic (Lewis 2009, p. 212). A further important point to note is that worlds that are spatiotemporally isomorphic are also ipso facto observationally isomorphic. This is in virtue of the fact that, for Lewis, worlds are nothing more than spatiotemporal arrangements of objects that instantiate patterns of fundamental properties, and all else supervenes upon that base. But because fundamental properties, strictly speaking, are simply abstract objects, viz. transworld sets of individuals, which properties are instantiated by which objects is not something that is observable (for it is impossible to observe that an object is a member of a set). Of course, which property-roles are present in a world is observable, but which property-roles are present is not determined by what properties fill those roles, and so this does supervenes upon the spatiotemporal arrangement of objects within a world. ${ }^{4}$

It is now easy to see how Humility follows from M1. Imagine that we have reached a stage where our physics is complete and make the confident assumption that its theoretical terms refer to unique properties that occupy the fundamental property roles that it describes (and thus that the actual world W@ is nomologically well-behaved). If this assumption is correct, then M1 tells us that there are worlds that are nomologically and observationally isomorphic with W@. The being nomologically and observationally isomorphic with relation is an equivalence relation and so partitions the set of all worlds into equivalence classes of worlds that are nomologically and observationally isomorphic with each other. Consider the class $C$ of worlds nomologically and observationally isomorphic with $W @$. For each one of us there is an observer in each world in $C$ to whom everything seems just as it does to each one of us. And in each member of $C$, those observers that correspond to us have a physical theory that looks just like ours, and they use the same theoretical terms as we do. And because each member of $C$ is also well-behaved, so the theoretical terms used by the observers in each member of $C$ refer to properties that uniquely occupy the fundamental roles that their physics describes. However, in each member of $C$ the theoretical terms used there refer to distinct properties from those that we refer to in $W @$ using the same terms. Now, consider an observer $o$ taken from some member of $C$. Whichever world $o$ comes from, $o$ has an observational duplicate in each of the other worlds in $C$, so $o$ has no way of telling, even in principle, which member of $C$ she inhabits. Thus $o$ cannot know which properties her theoretical terms refer to. However, if M1 is true, o's confident assumption that her world is well-behaved is not undermined, for on that assumption

Footnote 3 continued

However, in what follows I have found it useful to refer to observationally isomorphic worlds that contain no observers at all. On the weaker definition it is trivially true that any two worlds that contain no observers are observationally isomorphic_-and this won't do. So I stick with the stronger, but perhaps slightly less clear, definition.

${ }^{4}$ Lewis thus makes structuralist assumptions that some find implausible (see, e.g. Leuenberger 2010). My topic here, however, is Lewisian quidditism, so in what follows I proceed on Lewis's assumption that these structuralist assumptions are true. 
$o$ is justified in believing that her ignorance regarding which world she inhabits extends only as far as those worlds in $C$. So $o$ can know that, whichever world in $C$ she inhabits, her theoretical terms do refer to unique properties that occupy the roles that her physics describes. So if $o$ assumes that her world is well-behaved, and if M1 is true, $o$ is Humble. Furthermore, if M1 is true, each one of us is in $o$ 's position, and so each one of us is Humble too.

\section{Lewis's presentation of the permutation argument}

Lewis asks us to assume that we have the true and complete theory $T @$ of $W @$. He thus asks us to assume that the realization formula of $T @$ is uniquely satisfied by some n-tuple of fundamental properties $N$, and so that the Ramsey sentence of $T @$ is true. Next, he assumes that at least two members of $N$ fall into many-membered categories. ${ }^{5}$ He then asks us to hold fixed any other members of $N$ and permute the rest within their categories. The result will be a distinct world $W^{*}$ in which the realization formula of $T @$ is uniquely satisfied by a distinct n-tuple of fundamental properties $N^{*}$. So, the very same Ramsey sentence that is true in $W @$ will be true in $W^{*}$, and $W^{*}$ will be spatiotemporally isomorphic with $W @$. In effect, the only difference between $W^{*}$ and $W @$ will be in the pattern of instantiation of fundamental properties, and so $W^{*}$ will be nomologically and observationally isomorphic withW@:

Suppose, for instance, that we start with the actual world, and we permute two fundamental monadic properties $F 1$ and $F 2$, these being the actual referents of the T-terms $t 1$ and $t 2$, leaving all else fixed. Exchange $F 1$ and $F 2$ throughout the world... Then $F 2$ will be found in exactly those places in space and time (or, more generally, in the pattern of instantiation of fundamental relations and magnitudes) that correspond to the places where $F 1$ was found originally; and vice versa. And the laws of nature governing $F 2$ in the permutation will be just the same as the laws governing $F 1$ originally...; and vice versa... (Lewis 2009, p. 208)

But two questions arise, says Lewis. 1. Do we really obtain a genuine possibility by permutation? 2. Even if the possibility we obtain really is a genuine one, is it really a different possibility from the one we started with? If one is a Humean quidditist, the answer to both questions is Yes, says Lewis. The answer to the first is Yes, he says, because according to Humeanism possibility is governed by a principle of Recombination:

\footnotetext{
5 This assumption is true if, for example, there are at least two monadic properties. If we consider modern physics to be at least roughly correct this assumption is a safe one (it is true if modern physics is correct that negative charge and positive charge are fundamental, for instance). Lewis does, in fact, present a second argument for Humility called 'the Replacement Argument' that does not rely upon this assumption. The Replacement Argument instead relies upon the assumption that there are idle or alien properties of the same category as the properties that are actually instantiated. But aside from this the argument is identical in structure to the Permutation Argument, so I do not explicitly consider it here. Everything I say about the Permutation Argument can be applied mutatis mutandis to the Replacement Argument.
} 
We can take apart the distinct elements of a possibility and rearrange them. We can remove some of them altogether. We can reduplicate some or all of them. We can replace an element of one possibility with an element of another. When we do, since there is no necessary connection between distinct existences, the result will itself be a possibility. How much this means depends on what we take the distinct elements to be. Here, let us take them to include not only spatiotemporal parts, but also abstract parts-specifically, the fundamental properties. (Lewis 2009, pp. 208-209)

And the answer to the second question is Yes, says Lewis, because this is just what quidditism is. It is the thesis that possibilities can differ in just this way:

Combinatorialism tells us that possibility is preserved under permutations of items-at least if they are items from the same category. If it is possible that $-\mathrm{A}-\mathrm{B}-\mathrm{C}-$, and if $\mathrm{A}, \mathrm{B}$, and $\mathrm{C}$, are, say, all-or-nothing monadic properties, then it is also possible that $-\mathrm{C}-\mathrm{A}-\mathrm{B}-$. The actual realization of $T$ is a possible realization; we permute items within more-than-one-membered categories; and what we get is also a possible realization of $T$. But is it different from the one we had before? ... Quidditism is the premise that tells us that the permutation is indeed a different possibility... Two different possibilities can differ just by a permutation of fundamental properties. (Lewis 2009, p. 209)

Such is Lewis's presentation of the Permutation Argument. It is supposed to establish that if we are Humean quidditists we can start from the actual world and, on the assumption that our theoretical terms refer (and that some properties fall into many-membered categories), we can get to distinct nomologically and observationally isomorphic worlds by Recombination. So, it is supposed to establish that M1 follows from Humean quidditism.

\section{Problems with Lewis's presentation}

In the quoted passages above Lewis says that according to the principle of Recombination 'we can take apart the distinct elements of a possibility and rearrange them' and that how much this means 'depends on what we take the distinct elements to be'. He then says that in giving his argument he is taking properties themselves to be 'parts' of possibilities. But this is in tension with his official view that properties are transworld sets of individuals. It is easy enough to see how we can take apart properties from a possibility if they are supposed to be genuine parts of worlds-i.e. entities that are in some sense independent of the individuals that instantiate them (e.g. universals or tropes). But how this is supposed to work if properties are transworld sets (which are not genuine parts of worlds and are in no way independent on the individuals that instantiate them) is, at best, mysterious. Moreover, if we turn to what Lewis says elsewhere about the principle of Recombination it seems that on his view it is an essentially first-order principle (i.e. one that can apply only to individuals), and that as a consequence it can have nothing whatsoever to say about how to permute properties by Recombination. All 
of this suggests that, in fact, Lewis ought to reject the Permutation Argument, at least as he states it.

In order to draw out these difficulties more fully let us first consider a toy example that illustrates how the Permutation Argument, as stated, might be supposed to work.

Consider a world $W 1$ containing nothing except four individuals $a, b, c$ and $d$. For the sake of simplicity assume here (and in all later examples) that these individuals are mereologically simple. Now suppose that $W 1$ is a nomologically well-behaved world and so that each property role is uniquely filled by one and only one property. And further suppose that we identify a complete theory for Wl in which two fundamental (monadic) property roles are specified. As $W 1$ is nomologically wellbehaved, then we can form the names ' $P$ ' and ' $N$ ' for whatever property occupies each of these roles in $W l$, and our theory will tell us precisely which individuals instantiate $P$ and $N$. Finally, let us suppose that it is $a$ and $b$ that instantiate $P$, and $c$ and $d$ that instantiate $N$, and that they are arranged as follows:

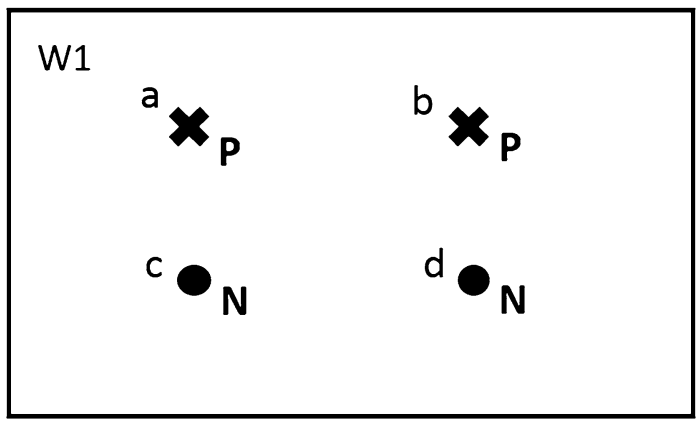

In this diagram the property-roles that $P$ and $N$ play in $\mathrm{W} 1$ are represented by the shape of the individuals that possess them-so $P$ occupies what we might call 'the cross-role', and $N$ 'the dot-role'.

Now, in $W 1$ the property $P$ uniquely occupies the cross-role, and $N$ uniquely occupies the dot-role. We may suppose that it is a law in Wl that everything that instantiates $P$ is above everything that instantiates $N$, and so the true and complete theory $T 1$ of $W 1$ will say as much. $T 1$ will say a bunch of others things too, but for simplicity let's suppose it does not. Ignoring all the other things that $T 1$ says, then, we can take the postulate of $T 1$ to be the simple sentence "All things that instantiate $P$ are above all things that instantiate $N$ '. By replacing ' $P$ ' and ' $N$ ' by the variables ' $x_{1}$ ' and ' $x_{2}$ ' we get the realization formula of $T 1$ : "All things that instantiate $x_{1}$ are above all things that instantiate $x_{2}$." The pair $\langle P, N\rangle$ uniquely satisfies this formula, and so is the actual realization of $T 1$. By prefixing the postulate with existential quantifiers we get the Ramsey sentence of $T 1$, "For some $x 1$, some $x 2$ (All things that instantiate $x 1$ are above all things that instantiate $x 2)$ ", which is true.

Now we apply the Humean principle of Recombination to permute $P$ and $N$. In order to do so we have to deinstantiate $P$ from all those individuals in $W 1$ that instantiate it (i.e. $a$ and $b$ ) and reinstantiate it in all those individuals that instantiate $N$ (i.e. $c$ and $d$ ), and de-instantiate $N$ from all those individuals that instantiate it 
$(c$ and $d$ ) and reinstantiate it in all those individuals that instantiate $P$ ( $a$ and $b$ ). In this way we get a world $W 2$ :

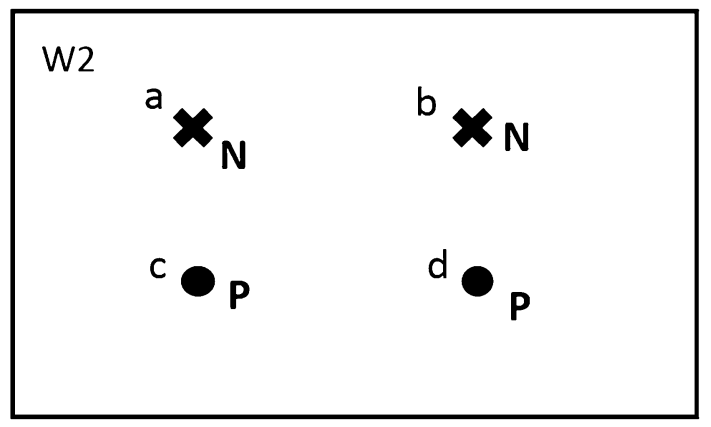

This world is exactly like $W 1$ except that $P$ now uniquely occupies the dot-role and $N$ uniquely occupies the cross-role. In particular, $W 2$ has the same pattern of distribution of individuals as $W 1$, so it is spatiotemporally isomorphic, and so observationally isomorphic. Quidditism is the thesis that worlds can differ in just this way, and so $W 2$ really is distinct from $W 1$. In $W 2 T 1$ is false because its postulate is: it is false that all things that instantiate $P$ are above all things that instantiate $N$. But the realization formula of $T 1$ is still uniquely satisfied in $W 2$ (now by $\langle N, P>$ rather than $\langle P, N>$ ) and so the Ramsey sentence of $T 1$ is still true in $W 2$. So $W 2$ is non-identical but nomologically isomorphic with $W 1$. So the analogue of M1 for $W 1$ is true. This is a toy example. But it is clear how to apply the same argument to any well-behaved world that has at least two monadic fundamental properties within it. So it is clear how to apply it to the actual world. So it is clear how the argument is supposed to establish the truth of M1 itself.

Suppose one is a Humean quidditist who believes that properties are genuine parts of worlds (e.g. that properties are universals or tropes). Then it is easy to see why one might consider the toy example to be a good illustration of the Permutation Argument in action. As Lewis says, the Humean principle of Recombination tells us that we can take apart and rearrange distinct elements of worlds, and that what this means depends on what we take the distinct elements to be. If one takes properties to be genuine parts of worlds then one might think that Recombination can be applied to them in a way that justifies the move made above from $W 1$ to $W 2$. One might conceive of Recombination as a procedure whereby one pulls out properties from the objects that instantiate them like pins from a pin-cushion, and then sticks them back in elsewhere. So to get from $W 1$ to $W 2$ all one does is pull out $P$ from $a$ and $b, N$ from $c$ and $d$, then stick $P$ back into $c$ and $d$, and $N$ back into $a$ and $b$. All this is fine, for those who conceive of properties in this way. But the problem is that Lewis does not conceive of properties in this way. And if we conceive of properties as Lewis does, we cannot cash out Recombination as we have above, and it seems that we should in fact deny outright that the toy example is a good example of the Permutation Argument in action.

On Lewis's view properties are transworld sets of individuals. Every set of individuals is a property and every property is a set of individuals. The fundamental 
properties are a select minority of those sets that are, somehow or other, special (they "glow in the dark" as Stephen Barker has put it to me in conversation). Now ask: What does it mean to permute a transworld set by Recombination? How is one supposed to take transworld sets apart within a world and rearrange them? These questions are puzzling because, conceived of in this way, properties are not genuine parts of worlds at all. On this view we cannot conceive of Recombination as we did above. No sense can be made of the idea that we can pull out transworld sets from the objects that instantiate them and stick them back in elsewhere. Conceived of as universals or tropes properties can be thought of as being like pins in a pin-cushion, but conceived of as transworld sets they cannot.

Consider what Lewis himself says about the distinction between universals and trans-world sets:

Universals and properties [i.e. properties conceived of as transworld sets] differ in two principled ways. The first difference concerns their instantiation. A universal is supposed to be wholly present wherever it is instantiated. It is a constituent part (though not a spatiotemporal part) of each particular that has it. A property, by contrast, is spread around. The property of being a donkey is partly present wherever there is a donkey, in this or any other world. Far from the property being part of the donkey, it is closer to the truth to say that the donkey is part of the property. But the precise truth, rather, is to say that the donkey is a member of the property. (Lewis 1983, pp. 344-355)

This is why properties conceived of as transworld sets are not genuine parts of worlds at all. The only genuine parts of worlds are the objects that fall into them. So it is difficult to see how Recombination is supposed to apply to properties on Lewis's view. Consider a concrete example. Suppose we wish to permute negative and positive charge within the actual world by Recombination. Negative charge, according to Lewis, is the special transworld set of individuals that has as its members all of the this-worldly and otherworldly negatively charged individuals. Positive charge is a similar special set. So how exactly do we permute these things by Recombination? We cannot permute the sets (what does it even mean to say this?). So what do we do? Do we permute their thisworldly members for one another? No, because this is to permute the individuals that have the property and not the properties themselves. Might this not amount to the same thing? Certainly not—at least not on Lewis's view. Lewis is an anti-haecceitist, so on his view the individuals within a world are just the indiscernible hooks that 'the [fundamental] properties and relations are hanging on'. If we merely permute individuals by Recombination what we end up with when we've finished rearranging things is the same possibility that we started with (see Lewis 1994, p474). So again, what do we do? The question is puzzling. ${ }^{6}$

\footnotetext{
6 The genesis of this paper was precisely a sense of puzzlement that arose in me when I first read Lewis's argument. I am here outlining the questions that lead to that perplexity. I remained perplexed for quite some time. I read around and asked others for help. But there is very little written on the Permutation Argument. And most of those I spoke to, once the questions here were raised, became as perplexed as I was.

There are two extant discussions of the Permutation Argument. And there is one person who seemed not to be perplexed by my questions:
} 
There are are further difficulties. Even if we suppose that we can make sense of permuting things by Recombination that are not genuine parts of worlds, there are serious difficulties with the argument from the Lewisian perspective. In the toy example we did not permute the individuals in $W l$ by Recombination, of course. We permuted the properties as we were supposed to. And when we'd finished we ended up with $W 2$, which was supposed to be a distinct world from $W 1$ in virtue of its properties being in different places. If quidditism is true and properties are genuine entities like universals or tropes it is easy to see how this can be so-the worlds differ by having different entities in different places. But if the only genuine parts of $W 1$ and $W 2$ are the individuals within them, then how are $W 1$ and $W 2$ different from one another? The worlds have exactly the same (numerically identical) parts arranged in exactly the same way. In fact, for Lewis, this question is not even a sensible one. No two worlds ever have numerically identical parts because individuals are world-bound. What this means is that, on Lewis's view, if the argument given is to be at all sensible, it must be that $W 2$ is described incorrectly above. $W 2$ can't be just like $W 1$, except that the properties have swapped places. $W 2$ must contain distinct individuals from $W 1$ too. But this means that when we permute

\section{Footnote 6 continued}

Dustin Locke discusses the Permutation Argument in his (2012). At one point he seems to recognise that the argument is problematic from the Lewisian perspective. In the course of his discussion he asks the salient questions: 'what precisely is being recombined when we move between possibilities in Lewis's argument?' and 'how, exactly, are they being recombined?' But he then goes on to say that from the Lewisian perspective properties are being permuted 'with respect to their spatiotemporal distributions' and that Lewis has 'a principle that entails that possibility is preserved under permutation of properties with respect to their spatiotemporal distributions.' (pp. 360-361) As we will see, I think that this is in fact true. But it is by no means obvious that it is true, for precisely the reasons I am giving here. And in fact, as will become clear, it takes a lot of work to see that it is true. But Locke says no more than this. So what Locke says does not really help us to understand the Permutation Argument.

Stephan Leuenberger offers a reconstruction of the Permutation Argument in his (2010) (in fact, he reconstructs the Replacement Argument, but he acknowledges that what his formulation applies mutatis mutandis to the Permutation Argument). But Leuenberger's argument does not look much like Lewis's to me. Indeed, according to Leuenberger, quidditism is a conclusion of the Permuation Argument. (pp. 330-331) This, I think, is mistaken. Lewis is quite clear that quidditism is a premise in the argument. So whether or not the argument Leuenberger gives is a good one, I don't think it can plausibly be considered to be the one Lewis intends to present.

Another prominent philosopher (who I shall not name) suggested to me in conversation that what Lewis has in mind is that we permute the names of fundamental properties rather than the properties themselves. But this just can't be right. Firstly, if all we were doing were permuting names within our term for the n-tuple that realizes T@ (or in the toy example the names ' $\mathrm{P}$ ' and ' $\mathrm{N}$ ' within ' $<\mathrm{P}, \mathrm{N}>$ ') then all we would be doing is relabeling the properties we've already got, and there would be no way to end up with a distinct possibility at the end. (I can call my mother 'father', but it doesn't follow from my doing so that she might have been my father.) But secondly, and more importantly, it is simply not plausible to think that Lewis had some sort of semantic procedure in mind. Recombination is supposed to apply at the metaphysical level and everything Lewis says suggests this. When we apply Recombination we are not merely supposed to be switching names about within our term for the realization of $T @$. Rather, we are supposed to be pulling apart possibilities themselves and rearranging them. Lewis says that we permute $\mathrm{A}$, $\mathrm{B}$ and $\mathrm{C}$ within $<-\mathrm{A}-\mathrm{B}-\mathrm{C}->$ to get $<-\mathrm{C}-\mathrm{A}-\mathrm{B}->$, not ' $\mathrm{A}$ ', ' $\mathrm{B}$ ' and ' $\mathrm{C}$ ' within ' $<-\mathrm{A}-\mathrm{B}-\mathrm{C}->$ ' to get ' $<-$ C-A-B->'. 
fundamental properties by Recombination we must somehow end up with a world that contains distinct individuals from the world with which we started. But how exactly does this happen if all we are supposed to be doing is permuting properties whilst leaving the individuals where they are?

The problems above arise when we try to apply Recombination in the way described in order to permute properties on Lewis's view that properties are transworld sets. In short, one simply cannot do so. This alone is sufficient for thinking that Lewis should reject the Permutation Argument as it has been stated. But, to drive the nail into the coffin, consider that there are even places where Lewis himself explicitly denies that Recombination applies to properties in the way in which it was applied it in the toy example. Consider, for example, the following passage from 'Armstrong on Combinatorialism' (that comes under the heading 'What is There to Recombine?'):

Almost anyone could be some kind of combinatorialist. But Armstrong's own brand of combinatorialism depends heavily upon his account of the constitution of this world-the world, if he is right, the one and only world. What possibilities we get by recombining the elements of the world depends on what those elements are. If we think the elements are point particles, we can use recombination to generate all manner of weird and wonderful rearrangements of particles, but we cannot get new kinds of particles... Whereas if we think with Armstrong that even the smallest particles are 'layercakes' made of a particular substratum plus several universals, one for mass and one for charge and so on, then indeed we can get new kinds of particles by recombining the layers of the cake. (Lewis 1999, p. 199)

Here Lewis is talking about the possibility of obtaining (by Recombination from the actual world) particles that instantiate alien combinations of properties, e.g. a particle that instantiates both mass and charge. He thinks that Armstrong has the resources to do so, but that he does not. But Lewis is quite clear here: If one does not believe that properties are genuine entities then Recombination simply does not apply to them.

So what does all of this show? Does it show that the Permutation Argument is itself flawed from a Lewisian perspective? No. It shows that Lewis states the argument loosely in 'Ramseyan Humility'. The main looseness is with his talk of 'permuting properties', and of 'pulling apart and rearranging possibilities'. Speaking in these loose terms is perhaps useful heuristically, when considering what possibilities there are. But however useful it may be, it obscures the real principle of Recombination, and it obscures our understanding of the Permutation Argument. It makes Recombination sound as if it is some sort of procedure that we perform. But of course it is no such thing. Even God cannot literally pull apart possibilities and rearrange them, so we have no chance.

Recombination is not a procedure. It is a statement about what possibilities there are. Lewis is right that the Humean principle of Recombination, together with quidditism, entails M1. But, in order to clearly understand how they do so, we must abandon Lewis's loose way of talking in 'Ramseyan Humility' and formulate Recombination more precisely. 


\section{The permutation argument correctly stated}

Lewis offers his own explicit (albeit rough) formulation of the principle of Recombination in On the Plurality of Worlds (Lewis 1986, p. 88). The question of how to formulate the principle more precisely has since received attention in the literature and there has been a gradual process of refinement resulting in the following statement of the principle:

REC: For any sequence $S 1$ of $n$ individuals $\langle x 1, x 2, x 3 \ldots>$ and any $n$-place spatiotemporal relation $R$, there exists a sequence $S 2$ of $n$ numerically distinct individuals $<y 1, y 2, y 3 \ldots>$ such that $S 2$ satisfies $R, y i$ duplicates $x i$, and the $y \mathrm{~s}$ form a maximal spatiotemporally related sum. ${ }^{7}$

So, does REC, together with quidditism, entail M1, given Lewis's view that fundamental properties are special transworld sets of individuals? At first sight one might remain somewhat puzzled. REC is a first-order principle that tells us, in effect, that if there are worlds containing certain individuals then there are worlds containing certain other individuals. Speaking loosely once more, it seems that all it allows us to do is to pull apart objects within possibilities and rearrange them. But it seems to say nothing whatsoever about properties. Moreover, REC appears to be a principle that is essentially first-order. The reference to duplicates within it appears to guarantee this, for the notion of duplication seems to make no sense when applied to transworld sets of individuals. ${ }^{8}$ So, it seems there is no way to reformulate the principle to obtain an analogous principle for properties (e.g. by replacing the firstorder quantifiers with second-order ones). Considerations such as these might lead one to think that a separate Humean principle of Recombination is needed for properties-perhaps a principle that can be initially stated in a rough manner as follows:

P-REC: Any object can instantiate or fail to instantiate any intrinsic property $\mathrm{P}$ without any further alteration in the distribution of intrinsic properties across the world.

\footnotetext{
${ }^{7}$ This formulation is equivalent to the one given by Darby and Watson in their excellent (2010) paper on the principle of Recombination (p. 444). See also Efird and Stoneham (2008) for discussion of the principle. Darby and Watson include a discussion of whether $\mathrm{n}$ can be set to 0 or not (concluding that it cannot) that is not relevant here and that I ignore. But for the sake of completeness, because I agree with their conclusion, take $\mathrm{n}$ here as ranging over all cardinals strictly higher than 0 . It is also worth noting that a sequence $S$ of $n$ individuals satisfies an n-place relation $R$ iff the members of $S$ stand in $R$ to one another in the order that they appear in the sequence. $\mathrm{n}$-adic spatiotemporal relations have $n$ ordered gaps in them that are occupied by $n$ individuals in a particular order. If John is to the left of Kevin then $<$ John, Kevin $>$ satisfies the to the left of relation but $<$ Kevin, John $>$ does not.

${ }^{8}$ For duplication, as Lewis defines it, seems to be essentially first-order. He defines it as follows: 'two things are qualitative duplicates if they have the same [fundamental] properties' (Lewis 1983 p. 356). It may be that we need to add a second clause to this definition as Lewis does in On the Plurality of Worlds: 'two things are duplicates iff (1) they have exactly the same [fundamental] properties, and (2) their parts can be put into correspondence in such a way that corresponding parts have exactly the same [fundamental] properties, and stand in the same [fundamental] relations.' (Lewis, 1986, p. 61) Lewis himself says this second clause may be redundant. But, at any rate, nothing hangs on taking this into account here.
} 
Of course, this formulation needs refinement too, but its basic content is clear enough. And indeed, it was a principle just like this that was tacitly relied upon in the toy example when we moved from $W 1$ to $W 2$. According to Humeans, monadic fundamental properties are intrinsic, so the properties $P$ and $N$ that filled the crossrole and the dot-role in the toy example are intrinsic. We might (as I did earlier) cash out the principle loosely by saying that we can pull out fundamental properties from individuals like pins from a pin-cushion and stick them back in elsewhere. But P-REC, suitably refined, gives the real content of this loose way of speaking.

P-REC is a principle that applies to properties regardless of whether they are universals, tropes, or sets. Moreover, it is plausible that any Humean is committed to something like P-REC. Humeanism is often stated, as I stated it at the very beginning of this paper, as being the view that 'there are no necessary connections between distinct existences'. But this is something of a misnomer. It misses an important aspect of Humeanism that is being emphasised here, namely, that there are no necessary connections between distinct instantiations of intrinsic properties either. For example, if $F$ and $G$ are fundamental (and so intrinsic) properties, $a$ is $F$, $b$ is $G$, and $a \neq b$, that $a$ instantiates $F$ does not entail that $b$ instantiates $G$, or vice versa. So there is no logical dependency between the instantiation of $F$ by $a$ and $G$ by $b$. So any Humean ought to believe that if $F a \& G b$, then it could be that $\sim F a \& G b$, it could be that $F a \& \sim G b$, and indeed, it could be that $G a \& F b$. Certainly Lewis himself seems to endorse something like P-REC. Consider the following passage from 'A World of Truthmakers?':

Possibility is 'combinatorial': there are two possibilities for whether or not Melbourne exists, two possibilities for whether or not Sydney exists, and either of the two possibilities for Melbourne can be combined with either of the two possibilities for Sydney. Likewise, possibilities about the intrinsic natures of distinct things are independent: Melbourne could be flat and Sydney could be hilly, or Sydney could be flat and Melbourne hilly, or both could be flat, or both could be hilly. And, likewise, whether Melbourne is flat or hilly is independent of whether Sydney does or does not exist: again, four different cases are possible. Or so we would all think. (Lewis 1999, 216)

One strategy for showing that M1 follows from Humean quidditism would thus be to go through the refining process with P-REC in order to formulate a principle that is acceptable given Lewis's views and that, together with quidditism, entails M1. That would work. But it's not worth the toil. We have no need to go through the refining process. Why not? Because we already have the finished product. It is none other than REC itself. I said that REC seems to say nothing whatsoever about properties. But in fact, it does. Despite REC being an essentially first-order principle, it does everything that we want P-REC to do, and together with quidditism, it does entail M1. To see how, consider our toy example once more, starting with W1: 


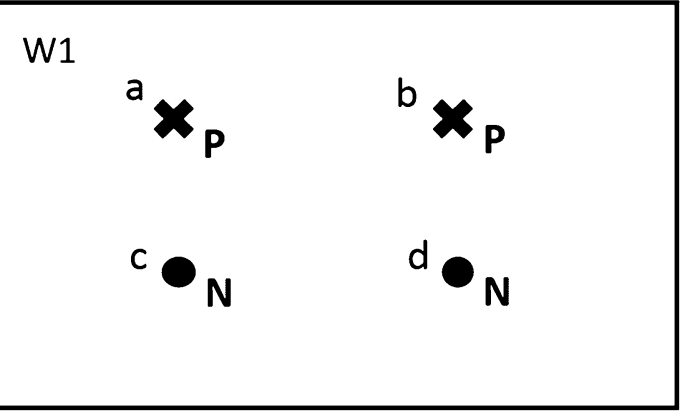

$a, b, c$ and $d$ stand in a particular four-place spatiotemporal relation in W1. Call this relation $S$. In $W l$ the sequence $\langle a, b, c, d\rangle$ satisfies $S$. But now consider the sequence of objects $\langle c, d, a, b\rangle$. REC tells us that there exists a sequence of numerically distinct objects $<c^{*}, d^{*}, a^{*}, b^{*}>$ that satisfies $S$ and is such that $c^{*}$ duplicates $c, d^{*}$ duplicates $d, a^{*}$ duplicates $a, b^{*}$ duplicates $b$, and the members of this sequence form a maximal spatiotemporal sum. In other words, it tells us that there is a world $W 2 *$ which is spatiotemporally isomorphic with $W 1$ but in which the objects $c *$, $d^{*}, a^{*}$ and $b^{*}$ are arranged just as $a, b, c$ and $d$ are in Wl (i.e. in that order). Moreover, because spatiotemporally isomorphic worlds are observationally isomorphic on Lewis's view, $W 2 *$ will be observationally isomorphic with $W 1$. Moreover, because which property-roles are present in a world supervenes on the spatiotemporal arrangements of objects within it, $W 2 *$ is a world in which the property-roles do not themselves switch places. But now remember that, for Lewis, duplication is a matter of sharing fundamental properties, i.e. that $x$ is a duplicate of $y$ iff $x$ has all and only the fundamental properties that $y$ has. So when we duplicate an object the fundamental properties of the original object get dragged along even though the property-roles stay behind. So in $W 2^{*} c^{*}, d^{*}, a^{*}$, and $b^{*}$ have all and only the fundamental properties of $W 1$ 's $c, d, a$, and $b$ respectively. Thus we can correctly represent $W 2 *$ as follows:

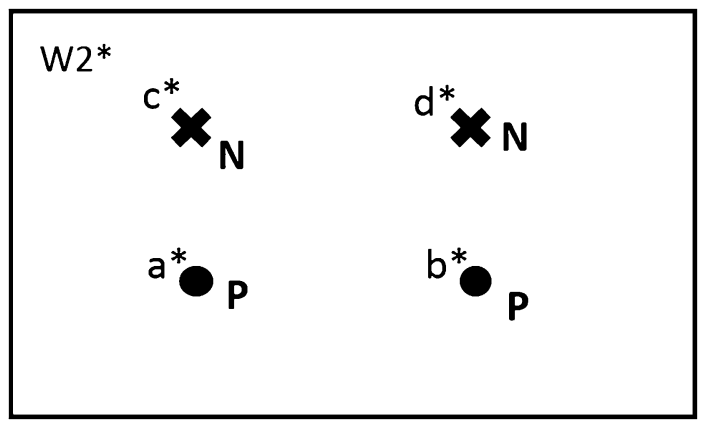

$W 2 *$ has the same pattern of distribution of individuals as $W 1$, but $N$ now uniquely occupies the cross-role and $P$ uniquely occupies the dot-role. ${ }^{9}$ Quidditism is the

\footnotetext{
9 We may also take $c *$ in $W 2 *$ as being a counterpart of $a$ in $W 1$. Then $W 2 *$ represents the possibility of $a$ being $N$ (mutatis mutandis for $d^{*}, a^{*}$, and $b^{*}$ in $W 2^{*}$ and $b, c$ and $d$ in $W 1$ ). But this is not essential. The
} 
thesis that worlds can differ in just this way, and so $W 2 *$ really is distinct from $W 1$. The true and complete theory $T 1$ of $W 1$ is false because its postulate is: it is false that all things that are $P$ are above all things that are $N$. But the realization formula of $T 1$ is uniquely satisfied (by $\langle N, P>$ ) and so the Ramsey sentence of $T 1$ is still true. Thus, as well as being observationally isomorphic with $W 1, W 2 *$ is nomologically isomorphic with $W 1$ too. So the analogue of M1 for $W 1$ is true on Lewis's view. Once more it is clear how to apply the same argument to the actual world. So M1 is true on Lewis's view. ${ }^{10}$

\footnotetext{
Footnote 9 continued

mere existence of a possibility that is isomorphic with $W 1$ in the relevant senses, but in which the properties have 'swapped places' is sufficient to establish Humility-for that is all that is required for the truth of M1 given in Sect. 1.

${ }^{10}$ I have received, from a very competent philosopher, the following objection to the interpretation of Lewis that I am outlining (I leave the objector anonymous):
}

Just what sort of recombination principle is required by Lewis' argument. [As you do] a nice job of making clear, Armstrong's recombination principle will not do: that principle concerns recombination of parts of a world, and for Lewis, properties are not parts of worlds. What is needed is a principle about the distribution of properties, or a principle that entails such a principle. [You identify] just such a principle: REC. However, I wonder whether REC is really strong enough for Lewis. REC entails that there are possible worlds where duplicates of the individuals in our world are spatiotemporally arranged differently from how the duplicated individuals in our world are arranged. For simplicity, suppose that our world contains nothing but particles with both positive charge and mass. Now those particles are spatiotemporally arranged in such and such a way. REC thus entails that there is a world with duplicates of those particles and where those duplicates are arranged differently from how they are arranged in our world. In other words, REC entails that there is possible world where mass and positive charge are distributed in a way that differs from how they are distributed in our world. What it does not entail, however, is that there is a world where there are particles that have mass but lack positive charge. But this is surely something that Lewis's Humeanism will want to say is possible.

However, I think the response here is clear. This is something that Lewis explicitly denies. That is what the quote from Lewis's 'Armstrong on Combinatorialism' given above makes clear. His principle of Recombination does not allow us to infer that there is world in which there are particles that have mass but lack charge from the existence of a world in which particles have mass iff they have charge. If our world is a world of the latter kind, then an individual that has charge but not mass is, in Lewis's terminology, an alien individual, and Lewis sees no way for the those who maintain that only the actual world exists (i.e. actualists) to admit the possibility of alien individuals (cf. Lewis, 2009: 212-213). Of course, Lewis is no actualist, so even if our world is a world in which particles have mass iff they have charge, he does not have to declare worlds containing (alien) individuals that have mass but not charge impossible. All he requires is the extra assumption that it is possible for there be a world in which something instantiates mass but not charge. This assumption is independently plausible, and Lewis thinks it is true. But it does not follow from Lewis's principle of Recombination itself. Lewis's principle of Recombination tells us which worlds are possible given that some worlds are possible. But it does not, by itself, tell us everything there is to know about which worlds are possible. The fact that at least one very competent philosopher has objected in this way lends support to my belief that this aspect of Lewis's view is not well understood. 


\section{Diffidence}

So far I have shown how the permutation argument leads to Humility, i.e. by entailing that the following principle is true:

M1: If the actual world is nomologically well-behaved then there exist distinct worlds that are nomologically and observationally isomorphic with the actual world.

But now consider a further principle:

M2: If the actual world is nomologically well-behaved then there exist distinct worlds that are observationally isomorphic but nomologically deviant from the actual world.

If M2 is true then we are more than just Humble, we are Diffident, i.e. we cannot even know that our fundamental property terms refer. Suppose, as we did in Sect. 1, we make the confident assumption that the theoretical terms of physics do refer (and so that $W @$ is well-behaved). Now consider the class $C^{*}$ of worlds that are merely observationally isomorphic to W@. M2 tells us that some of these worlds are nomologically deviant from W@. But, for each one of us there is an observer in each world in $C^{*}$ to whom everything seems just as it does to each one of us. And in each member of $C^{*}$, the observers there have a physical theory that looks just like ours, and they use the same theoretical terms as we do, and everything seems to them just as it does to us. However, in those nomologically deviant members of $C^{*}$ the theoretical terms used by its inhabitants fail to refer because no property occupies the fundamental property roles described by their physics. Now consider an individual $o^{*}$ taken from some member of $C^{*}$. Again, whichever world $o^{*} \operatorname{comes}$ from, $o^{*}$ has an observational duplicate in each of the other worlds in $C^{*}$, so $o^{*}$ has no way of telling, even in principle, which member of $C^{*}$ she inhabits. And this, unlike Humility, does undermine $o^{*}$ 's confident assumption that her theoretical terms refer. So $o^{*}$ cannot know whether her theoretical terms refer or not. So $o^{*}$ is Diffident. Furthermore, if M2 is true, we are in $o^{*}$ 's position, and so we are Diffident too. ${ }^{11}$

\footnotetext{
${ }^{11}$ In his (2007) Alexander Bird also argues that quidditism entails a stronger form of scepticism than Humility. According to the form of scepticism that Bird thinks follows from quidditism we cannot know whether any fundamental property roles are uniquely occupied. He argues that quidditism entails that if the actual world is well-behaved then there are worlds observationally isomorphic to it where two or more fundamental properties occupy the very same role. Bird's argument is very different from the one I present here, and so is his conclusion. In what way do our conclusions differ? Call a world in which two or more fundamental properties occupy the same role a Bird-world. Then Bird's conclusion is that quidditism entails that if the actual world is well-behaved then there are Bird-worlds that are observationally isomorphic with it. No such Bird-world is well-behaved. But if the actual world is wellbehaved then no such Bird-world is nomologically deviant from the actual world either. This is because although the realization formula uniquely satisfied at the actual world (if it is well-behaved) is not uniquely satisfied in any such Bird-world, the Ramsey sentence true at the actual world is still true at all such Bird worlds, i.e. it is still true that some property occupies the fundamental property roles present in such Bird worlds. As a consequence of this Bird's conclusion can be resisted by any quidditist. Quidditists can consistently maintain that it is a de dicto necessary truth that two fundamental properties never occupy the same role. On this supposition the statement that the realization formula of a theory $\mathrm{T}$ is uniquely satisfied is materially equivalent to the statement that it is (merely) satisfied, and so materially
} 
Now that we have in place a good understanding of the permutation argument, it is in fact quite easy to see that there is a simple extension of it that entails M2. I utilise the toy example once more, starting with W1:

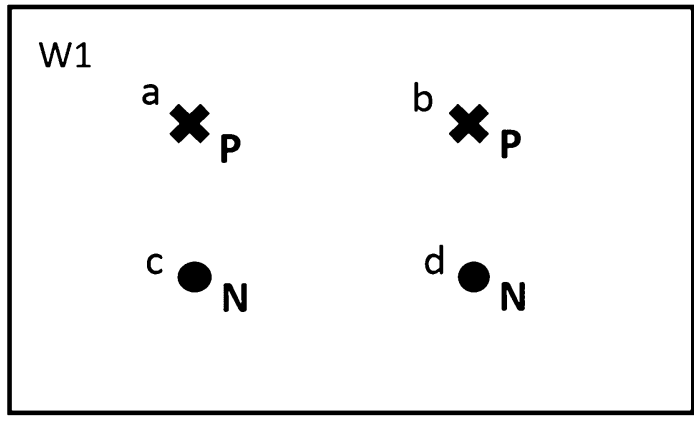

Consider once more that $\langle a, b, c, d\rangle$ satisfies the spatiotemporal relation $S$ in $W 1$. Now consider the sequence $\langle a, d, c, b>$. REC tells us that there exists a sequence of numerically distinct objects $<a^{*}, d^{*}, c^{*}, b^{*}>$ that satisfies $S$ and is such that $a^{*}$ duplicates $a, d^{*}$ duplicates $d, c^{*}$ duplicates $c, b^{*}$ duplicates $b$, and the members of the sequence form a maximal spatiotemporal sum. Again, the resulting world is spatiotemporally isomorphic with $W 1$, and so observationally isomorphic too. And again, in the resulting world the property-roles will not shift although the properties themselves will be dragged along with their duplicates. That is, REC tells us that there is a world W3 that can be represented as follows:

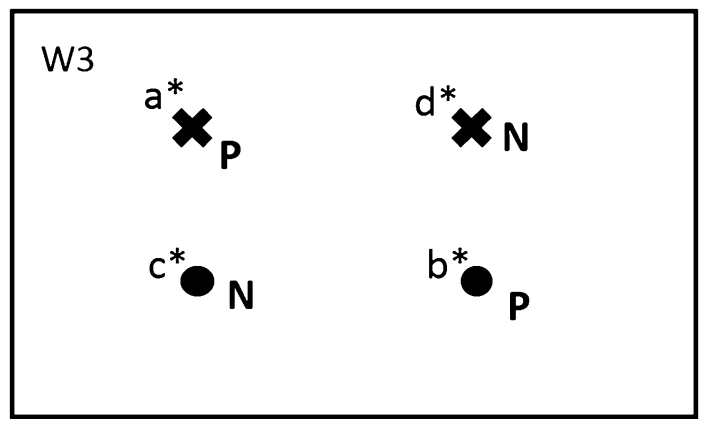

Footnote 11 continued

equivalent to the statement that the Ramsey sentence of $\mathrm{T}$ is true. So, on this supposition, any world that is observationally isomorphic to the actual world and is such that the Ramsey sentence that is true in the actual world is true there, is a world in which the property terms used in that world determinately refer. That quidditists have this way of resisting Bird's conclusion is pointed out by Noonan (2010). Noonan's defence against Bird's argument is no defence against mine, however, and does not enable Humean quidditists to resist my conclusion. Humean quidditists may maintain that it is a de dicto necessary truth that no two properties ever occupy the same role. But the assumption that the actual world is wellbehaved is still, on this view, the assumption that distinct fundamental properties occupy the cross-role and the dot-role in W1. And once this assumption is granted, the argument I will give goes through just the same. 
Although $W 3$ is spatiotemporally isomorphic with $W 1$, its parts fall into different special sets. Quidditism is the thesis that worlds can differ in just this way, so $W 3$ is really distinct from $W 1$. But although $W 3$ is observationally isomorphic with $W 1$, the realization formula of $T 1$ is not satisfied at all (uniquely or otherwise) and so the Ramsey sentence true in $W 1$ is false in $W 3$, i.e. it is not true that "For some $x 1$, some $x 2$ (All things that instantiate $x 1$ are above all things that instantiate $x 2$ )". So $W 3$ is nomologically deviant from $W 1$. So the analogue of M2 for W1 is true on Lewis's view. It is clear how to apply this argument to the actual world. So M2 is also true on Lewis's view. And so, therefore, is diffidence.

\section{Conclusion}

If we speak loosely we can speak of permuting properties by Recombination. But if we subscribe to Lewis's view it would be better to speak of replacing individuals with duplicates. To permute two fundamental (monadic) properties $F$ and $G$ in a well-behaved world we do not deinstantiate $F$ from all those individuals that instantiate it and reinstantiate it in all those individuals that instantiate $G$, and deinstantiate $G$ from all those individuals that instantiate it and reinstantiate it in all those individuals that instantiate $F$. Rather, we replace all those individuals that instantiate $F$ with duplicates of individuals that instantiate $G$, and replace all those individuals that instantiate $G$ with duplicates of individuals that instantiate $F$. If we do this we end up with a distinct world containing distinct individuals where $F$ and $G$ have swapped places with respect to their property-roles. This world will be observationally and nomologically isomorphic with the world we started with. The possibility of reaching such worlds from the actual world entails that Humility is true. But, by an extension of the argument we can replace just some of those individuals that instantiate $F$ with duplicates that instantiate $G$, and some of those individuals that instantiate $G$ with duplicates that instantiate $F$. If we do this we do not end up with a world in which $F$ and $G$ have swapped places with respect to their property-roles, but a world in which $F$ and $G$ no longer play any role at all. This world will be observationally isomorphic with the world we started with. The possibility of reaching such worlds from the actual world entails that Diffidence is true, and Diffidence is a much stronger form of scepticism than Humility.

Open Access This article is distributed under the terms of the Creative Commons Attribution 4.0 International License (http://creativecommons.org/licenses/by/4.0/), which permits unrestricted use, distribution, and reproduction in any medium, provided you give appropriate credit to the original author(s) and the source, provide a link to the Creative Commons license, and indicate if changes were made.

\section{References}

Bird, A. (2007). Nature's metaphysics: Laws and properties. Oxford: OUP.

Black, R. (2000). Against quidditism. Australasian Journal of Philosophy, 78(1), 87-104.

Darby, G., \& Watson, D. (2010). Lewis's principle of recombination: Reply to Efird and Stoneham. Dialectica, 64(3), 435-445. 
Efird, D., \& Stoneham, T. (2008). What is the principle of recombination? Dialectica, 62(4), $483-494$. Leuenberger, S. (2010). Humility and constraints on O-language. Philosophical Studies, 149(3), 327-354. Lewis, D. (1970). How to define theoretical terms. Journal of Philosophy, 67(13), 427-446.

Lewis, D. (1983). New work for a theory of universals. Australasian Journal of Philosophy, 61, 343-377. Lewis, D. (1986). On the plurality of worlds. Oxford: Blackwell.

Lewis, D. (1994). Humean supervenience debugged. Mind, 103(412), 473-490.

Lewis, D. (1999). Papers in metaphysics and epistemology. CUP: Cambridge.

Lewis, D. (2009). Ramseyan humility. In D. Braddon-Mitchell \& R. Nola (Eds.), Conceptual analysis and philosophical naturalism. Cambridge, MA: MIT Press.

Locke, D. (2012). Quidditism without quiddities. Philosophical Studies, 160(3), 345-363.

Noonan, H. W. (2010). Bird against the humeans. Ratio, 23(1), 73-86.

Sellars, W. (1948). Concepts as involving laws and inconceivable without them. Philosophy of Science, 15, 287-315. 